\title{
Reduction of myocardial infarct size by neutrophil depletion: Effect of duration of occlusion
}

\begin{abstract}
Experiments were performed in the dog to examine the effects of neutropenia on ultimate infarct size resulting from short (90 minutes) or prolonged (4 hours) circumflex coronary artery occlusion. Sheep antiserum to canine neutrophils was used to produce neutropenia. Control animals received nonimmune serum. Neutrophil infiltration into myocardial infarcts was examined using histopathologic techniques and a semiquantitative scoring system. In 90 -minute occlusions with 24-hour reperfusion, neutropenia was associated with the development of significantly smaller infarcts: normopenic group, $43.2 \% \pm 3.3 \%(n=7)$ vs. neutropenic group, $26.6 \%+3.7 \%$ $(n=10)$ of the area at risk, $\bar{X} \pm S E M$. However, in 4-hour occiusion with 6-hour reperfusion experiments, the tendency of neutrophil depletion to reduce infarct size did not reach statistical significance $(46.4 \% \pm 7.2 \%$ v8. $31.5 \% \pm 6.0 \%$ of the area at risk, normopenic vs. neutropenic) despite differences in neutrophil infiltration into the reperfused region. The observed differences in ultimate infarct size could not be attributed to differences in myocardial oxygen consumption. The results suggest that a significant amount of myocardial infarction induced by a limited duration of coronary artery occlusion followed by reperfusion is neutrophil dependent and appears to be less important in determining the fate of myocardium subjected to more prolonged periods of ischemia followed by reperfusion. (AM HeART J 1986;112:682.)
\end{abstract}

Stanley R. Jolly, Ph.D., William J. Kane, B.S., Bruce G. Hook, B.S., Gerald D. Abrams, M.D., Steven L. Kunkel, Ph.D., and

Benedict R. Lucchesi, Ph.D., M.D. Ann Arbor, Mich.

Previous studies from our laboratory have implicated a polymorphonuclear neutrophil (PMN)mediated inflammatory response in the development of myocardial infarcts after coronary artery occlusion (90 minutes) followed by reperfusion. ${ }^{1.3}$ Reduction of circulating PMNs in the dog with a rabbit-derived antiserum resulted in smaller myocardial infarcts. ${ }^{1}$ Other investigators using antiinflammatory agents and neutrophil antisera have reached similar conclusions regarding the deleterious effects of "early neutrophil entry" in myocardial regions subjected to ischemia and reperfusion. ${ }^{4-6}$ Earlier findings regarding neutrophil infiltration

From the Departments of Pharmacology and Pathology, The University of Michigan Medical School, Ann Arbor, Mich.

Supported by grants from the National Institutes of Health (HL-19782-06) and the American Heart Association of Michigan.

Received for publication Sept. 30, 1985; revision received Jan. 17, 1986; accepted Feb. 20, 1986

Reprint request: Benedict R. Lucchesi, Ph.D., M.D., Department of Pharmacology, M7423 Medical Science Bldg. I, The University of Michigan Medical School, Ann Arbor, MI 48109. have been extended in the present study using a sheep-derived antiserum and a longer period of reperfusion. A second experiment evaluated the relative importance of $\mathrm{PMN}$-mediated inflammation in the context of a more prolonged period of ischemia followed by reperfusion.

\section{METHODS}

Occlusion/reperfusion model of myocardial infarc. tion. Ischemic myocardial injury was produced in dogs using techniques detailed previously ${ }^{3,7}$ Male mongrel dogs (10 to $15 \mathrm{~kg}$ ) were anesthetized with pentobarbital sodium, $30 \mathrm{mg} / \mathrm{kg}$ intravenously, intubated and ventilated with room air via a Harvard respirator (Harvard Apparatus, Millis, Mass.). Catheters for drug infusion and arterial pressure measurement were implanted in the left jugular vein and left carotid artery. A thoracotomy was performed at the fifth left intercostal space. The heart was suspended in a pericardial cradle and the left circumflex coronary artery (LCCA) was isolated distal to its atrial branch and proximal to any major ventricular branches. An electromagnetic flow probe and a micrometer-driven coronary occluder $^{8}$ were placed on the LCCA. ECG limb lead II and phasic arterial pressure were recorded continuously on a 
EXPERIMENTAL PROTOCOL

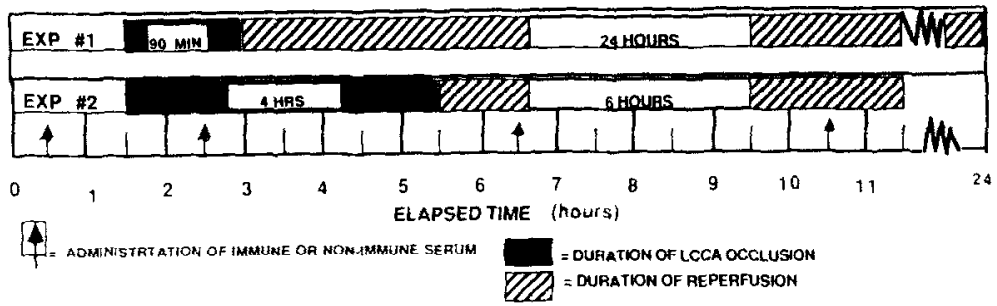

Fig. 1. Plan of protocol for experiments No. 1 and No. 2 are shown with time intervals for each intervention. Arrows indicate time of administration of neutrophil antiserum or of nonimmune serum. The durations of left circumflex coronary artery occlusion (LCCA) and reperfusion are shown as solid black and hatched horizontal bars, respectively. Note that protocol for experiment No. 1 terminated 24 hours after initiation of LCCA reperfusion whereas the protocol for experiment No. 2 was completed 6 hours after reperfusion.

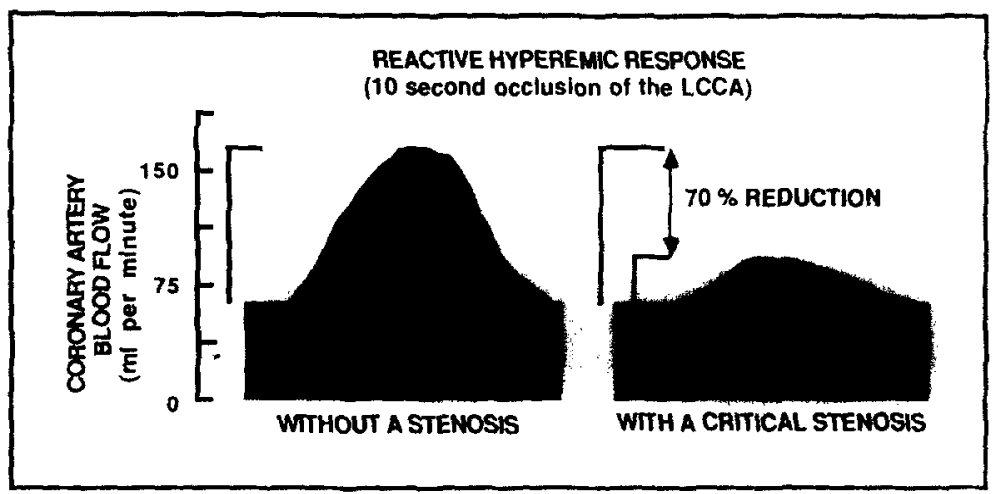

Fig. 2. Schematic representation demonstrating establishment of critical stenosis on left circumflex coronary artery $(L C C A)$. Reactive hyperemic response to 10-second occlusion of LCCA is shown on right before application of stenusis to coronary vessel. Reactive hyperemic response illustrated on left is obtained in presence of critical stenosis. Point of critical stenosis is defined as degree of constriction that produces a $70 \%$ reduction in reactive hyperemic response after release of a 10 -second occlusion of LCCA.

Grass model 7 polygraph (Grass Instrument Co., Quincy, Mass.).

Dogs were assigned randomly to treated or control groups (Fig. 1). Sixty minutes before occlusion, the dogs received $4 \mathrm{ml}$ of sheep-derived antineutrophil serum or nonimmune serum intravenously over 10 minutes. Active serum produced a brief decrease in systemic blood pressure, which returned to control within 10 minutes. Subsequent injections were without hemodynamic effect. Control measurements of hemodynamic parameters were obtained shortly before occlusion. The LCCA was occluded in two steps. First, the occluding device was adjusted carefully to obtain a degree of stenosis that did not affect resting coronary blood flow. The stenosis was adjusted to produce a $70 \%$ reduction from the control reactive hyperemic response that occurred after the release of a 10second occlusion of the vessel (Fig. 2). The LCCA then was occluded completely for 90 minutes (experiment No. 1) or for 4 hours (experiment No. 2). Antiarrhythmic drugs such as lidocaine were not used, since these compounds inde- pendently may affect myocardial infarct size. ${ }^{9}$ Animals that developed ventricular fibrillation during coronary artery occlusion or on reperfusion were defibrillated with low-power ( $15 \mathrm{~W}$ seconds) direct cardioversion by direct current (DC) (a total of three trials). If fibrillation reoccurred, the animal was excluded from further analysis. After the desired ischemic period, coronary flow was restored gradually over 30 minutes and the critical stenosis was maintained for an additional 10 minutes.

In experiment No. 1 infusion lines were exteriorized at the back of the neck, surgical wounds closed, and the animal allowed to recover from the surgical procedure. Three supplemental injections of antineutrophil serum were administered intravenously at 2,6 , and 10 hours after the initial treatment (Fig. 1). The next day, the ECG and arterial pressure were monitored with the dog resting quietly in a sling. Subsequently, the animals were reanesthetized, the original thoracotomy incision reopened to expose the heart, and the LCCA blood flow was recorded with an electromagnetic flow probe. The heart was fibril- 


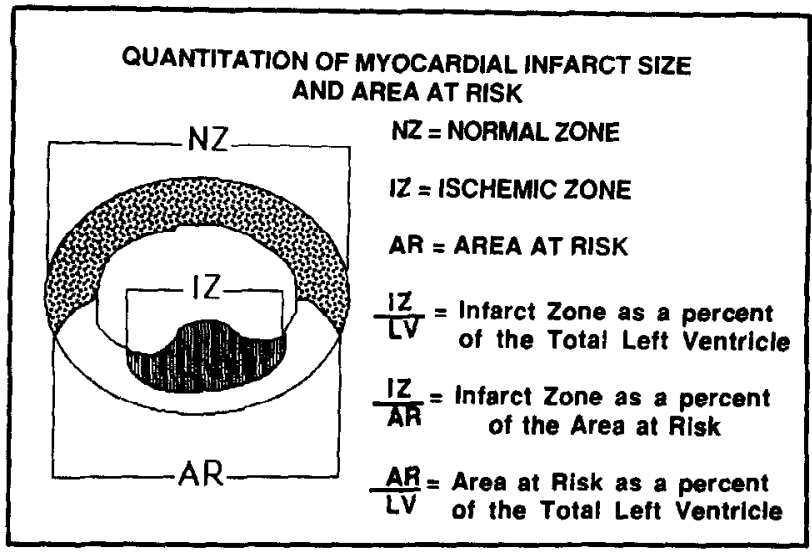

Fig. 3. Schematic representation of method used to quantitate infarcted zone (IZ), area at risk $(A R)$ and normal noninfarcted zone (NZ). (See text for details.)

lated electrically and removed rapidly for postmortem quantification of infarct size.

In experiment No. 2 (Fig. 1), an acute experimental protocol ( 4 hours of occlusion followed by 6 -hour reperfusion) was chosen because of an observed high mortality: 5 of 8 animals dying before the 24-hour end point. The surgical preparation, administration of antineutrophil serum, and the two-step occlusion of the LCCA were identical to the 90-minute occlusion protocol in experiment No. 1. After 4 hours of LCCA occlusion, reperfusion was accomplished by controlled restoration of coronary blood flow as described previously. A 6-hour reperfusion period was chosen to allow complete development of myocardial infarction as determined in previous studies from our laboratory. ${ }^{1,10}$ Supplemental injections of antineutrophil serum or nonimmune serum were made 2,6 and 10 hours after the initial treatment. This dosing schedule was selected on the basis of preliminary studies in which it was determined that the duration of the induced neutropenia would be maintained for 8 to 12 hours after a single dose. ${ }^{1}$ At the end of the experimental protocol, the heart was fibrillated electrically and removed for postmortem quantification of infarct size.

Postmortem quantification of infarct size. Myocardial infarct size was quantified using an in vitro dual perfusion technique described previously. ${ }^{11}$ Cannulae were inserted into the LCCA immediately distal to the site of LCCA occlusion and into the aorta above the coronary ostia. 'The LCCA coronary bed was perfused with $1.5 \%$ triphenyltetrazolium hydrochloride (TTC) in 20 millimole (mM) potassium phosphate buffer $\left(\mathrm{pH} 7.4,38^{\circ} \mathrm{C}\right.$ ). The aorta was perfused in a retrograde manner with $0.5 \%$ Evans blue stain. Because of its anatomic dependence on the LCCA for its blood supply, the area of the left ventricle at risk for infarction ( $A R$, Fig. 3 ) is demarcated by its lack of staining with Evans blue, which is delivered only to those regions supplied by the left anterior descending coronary artery and its branches and by the right coronary artery. The AR is perfused with TTC, an agent that turns into a

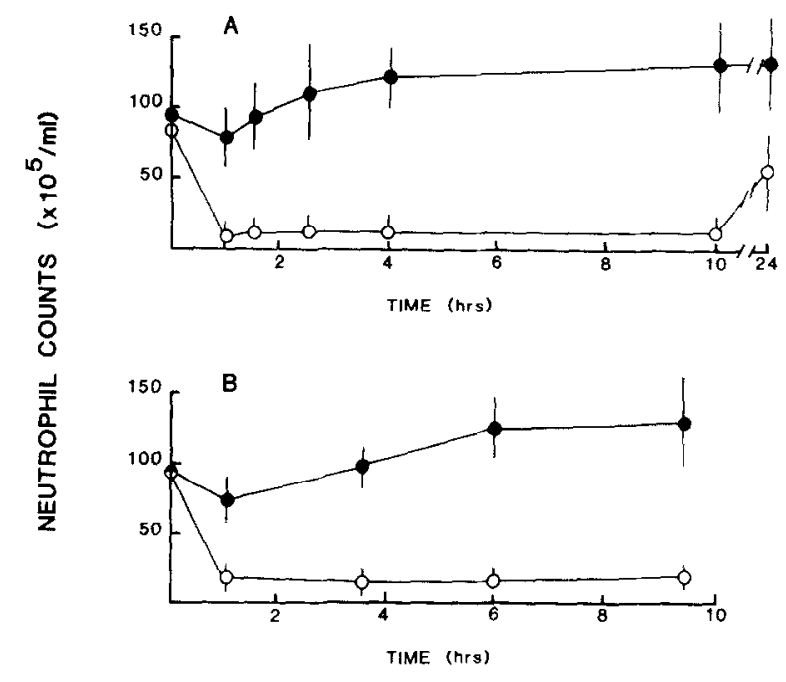

Fig. 4. Effects of neutrophil antiserum on circulating neutrophil counts. Neutrophils were counted manually in blood samples taken before serum administration ( $0 \mathrm{hr}$ ) and at intervals afterward. Each point represents mean value \pm SEM for each time from 90 -minute ischemia series (A) and 4-hour ischemia series (B) with closed circles $(\bullet)$ representing nonimmune serum group and open circles (o) representing antineutrophil serum group. Analysis of variance (Aov) followed by Duncan's multiple range test revealed no significant difference between groups at 0 hour. Neutrophil counts decreased significantly after treatment with antineutrophil serum on basis of between-groups comparison. Within-group comparison to 0 hour (2-way AOV) also revealed significant reduction in circulating neutrophils at all times except 24 hours (A) when $60 \%$ recovery of circulating neutrophils was noted. Neutrophil counts in nonimmune serum-treated groups tended to increase over course of experiment.

bright red formazan precipitate when it undergoes reduction in the presence of the dehydrogenase enzymes present in viable myocardial tissue. Failure of the perfused cells to reduce TTC is indicative as demarcating a region of irreversibly damaged myocardium. The method used for quantitating the extent of irreversibly damaged myocardium permits identification within the area at risk of the tissue that is irreversibly damaged (IZ, Fig. 3).

Tracings of each ventricular section and the associated regions as indicated by Evans blue staining and by the TTC reaction were made onto clear plastic sheets. The area of each of the demarcated regions in each ventricular section was quantitated by planimetry with the aid of an Apple IIe (Apple Computer Co., Cupertino, Calif.) microprocessor, appropriate software, and a digitizing board. In previous studies, planimetry has been given results identical to those obtained with the gravimetric method of analysis of the respective regions., ${ }^{31}$ The planimetric method of analysis was preferred for this study because it left the myocardial sections undisturbed for the subsequent histologic studies and semiquantitative assessment of neutrophil infiltration. The myocardial staining proce- 
Table I. Effect of neutrophil depletion on hemodynamic parameters during 90 minutes or 4 hours of regional myocardial ischemia followed by reperfusion*

\begin{tabular}{|c|c|c|c|c|c|c|}
\hline & \multirow[b]{2}{*}{ Control } & \multirow[b]{2}{*}{$\begin{array}{l}10 \text { minutes after } \\
\text { occlusion }\end{array}$} & \multirow[b]{2}{*}{$\begin{array}{l}15 \text { minutes before } \\
\text { reperfusion }\end{array}$} & \multicolumn{3}{|c|}{ Reperjusma } \\
\hline & & & & 45 minutes & 2 hrour: & Before killing \\
\hline \multicolumn{7}{|c|}{ Experiment 1: 90 -minute ischemic period } \\
\hline \multicolumn{7}{|c|}{ Nonimmune serum $(n=7)$} \\
\hline Heart rate (bpm) & $159 \pm 10$ & $159 \pm 8$ & $161 \pm 5$ & $154 \pm 5$ & $151 \div 4$ & $156+10$ \\
\hline $\mathrm{MAP}(\mathrm{mm} \mathrm{Hg})$ & $109 \pm 9$ & $88 \pm 7 \dagger$ & $96 \pm 7 \dagger$ & $95 \pm 6+$ & $99+6$ & $92 \pm 5^{+}$ \\
\hline Rate $\times$ pressure $\left(\times 10^{3}\right)$ & $19.0 \pm 1.8$ & $15.6 \pm 1.3 \dagger$ & $16.3 \pm 2.6$ & $16.3 \pm 1.1$ & $17.2 \pm 1.1$ & $16.9 \pm 2.0$ \\
\hline LCCA coronary flow $(\mathrm{ml} / \mathrm{min})$ & $18 \pm 1$ & 0 & 0 & $16 \pm 2$ & 0 & $10 \pm 3+$ \\
\hline $\mathrm{LV}$ end diastolic pressure $(\mathrm{mm} \mathrm{Hg}$ ) & $3 \pm 1$ & $6 \pm 1 \dagger$ & $5 \pm 1+$ & $8 \pm 1+$ & : & $12 \pm 2$ \\
\hline \multicolumn{7}{|l|}{ Neutrophil antiserum $(n=10)$} \\
\hline Heart rate (bpm) & $145 \pm 9$ & $144 \pm 9$ & $144 \pm 7$ & $146 \pm 7$ & $151=i$ & $146 \pm 9$ \\
\hline MAP (mm Hg) & $113 \pm 9$ & $95 \pm 8$ & $99 \pm 8 \dagger$ & $108 \pm 8$ & $116 \pm 10$ & $7 \pi \pm 9+\ddagger$ \\
\hline Rate $\times$ pressure $\left(\times 10^{3}\right)$ & $19.4 \pm 2.5$ & $16.5 \pm 2.2 \dagger$ & $16.7 \pm 1.7$ & $18.0 \pm 1.8$ & $20.2 \pm 2.2$ & $14.4 \pm 1.47$ \\
\hline LCCA coronary flow $(\mathrm{ml} / \mathrm{min})$ & $25 \pm 5$ & 0 & 0 & $27 \pm 5$ & 1 & $15 \pm 3+$ \\
\hline $\mathrm{LV}$ end-diastolic pressure $(\mathrm{mm} \mathrm{Hg})$ & $2 \pm 1$ & $5 \pm 1 \dagger$ & $6 \pm 1+$ & $6 \pm 1 \uparrow$ & i) & $7 \pm 1+\frac{1}{4}$ \\
\hline \multicolumn{7}{|c|}{ Experiment 2: 4-hour ischemic period } \\
\hline \multicolumn{7}{|c|}{ Nonimmune serum $(n=8)$} \\
\hline Heart rate $(\mathrm{bpm})$ & $162 \pm 11$ & $159 \pm 12$ & $171 \pm 9$ & $187 \pm 13 t$ & $197 \pm 134$ & $226+217$ \\
\hline MAP $(\mathrm{mm} \mathrm{Hg})$ & $126 \pm 4$ & $109 \pm 6 \dagger$ & $117 \pm 3$ & $105 \pm 5+$ & $107 \pm 87$ & $84 \div 74$ \\
\hline Rate $\times$ pressure $\left(\times 10^{3}\right)$ & $22.6 \pm 1.9$ & $19.1 \pm 2.0 \dagger$ & $21.6 \pm 1.0$ & $20.5 \pm 1.3$ & $22.6 \pm 1.6$ & $22.2+2.9$ \\
\hline LCCA coronary flow $(\mathrm{ml} / \mathrm{min})$ & $16 \pm 3$ & 0 & 0 & $11.1 \pm 1+$ & $11+1+$ & $7+1+$ \\
\hline $\mathrm{LV}$ end diastolic pressure $(\mathrm{mm} \mathrm{Hg})$ & $3 \pm 1$ & $6 \pm 1 \dagger$ & $7 \pm 1+$ & $- \pm 1 \dagger$ & $n \div 16$ & $11 \div 1+$ \\
\hline \multicolumn{7}{|l|}{ Neutrophil antiserum $(n=9)$} \\
\hline Heart rate (bpm) & $158 \pm 5$ & $153 \pm 6$ & $156 \pm 7$ & $178 \pm 10$ & $201=13 \%$ & $222 \pm 18+$ \\
\hline MAP (mm Hg) & $116 \pm 6$ & $99 \pm 6+$ & $99 \pm 3+t$ & $93 \pm 4 \dagger$ & $94 \pm 5+1$ & $71+5 \dagger$ \\
\hline Rate $\times$ pressure $\left(\times 10^{3}\right)$ & $20.8 \pm 1.5$ & $18.1 \pm 1.7 \dagger$ & $17.9 \pm 1.1 \dagger$ & $19.4 \pm 1.9$ & $22.2 \pm 2.1$ & $20.1=1.8$ \\
\hline LCCA coronary flow $(\mathrm{ml} / \mathrm{min})$ & $20 \pm 4$ & 0 & 0 & $13 \pm 2 \div$ & $15+3$ & $9+1+$ \\
\hline $\mathrm{LV}$ end diastolic pressure $(\mathrm{mm} \mathrm{Hg})$ & $3 \pm 1$ & $6 \pm 1 \dagger$ & $7 \pm 1+$ & $8 \pm 1 \dagger$ & $8 \pm 17$ & $g=1 \dagger$ \\
\hline
\end{tabular}

$* \overline{\mathrm{X}} \pm$ SEM is given. No differences among the four groups for hemodynamic parameters during the control period were observed. $t_{p}<0.05$ compared with control value.

$\ddagger p<0.05$ between control and depleted groups.

dure, tracing, and digitizing of the ventricular sections was performed by an individual unaware of the treatment protocol.

Histopathologic examination of myocardial tissue. The heart tissue sections were fixed in buffered $10 \%$ formalin. Two transmural tissue samples were excised from a midventricular myocardial section of each heart in a manner that resulted in cross sections of the area at risk and infarct with a small margin of nonischemic (blue stained) myocardium at either end of the tissue sample. Each tissue sample was embedded in paraffin, cut into 5 $\mu \mathrm{m}$ sections and stained with hematoxylin and eosin. In each experiment, light microscopy was used to compare the subendocardial center of the unstained (TTC) myocardium with the blue-stained nonischemic marginal tissue to confirm histologically the presence of irreversible tissue injury. A semiquantitative grading procedure was used to examine the extent of neutrophil infiltration into each area of infarct.' Briefly, a score of ++++ was assigned to the myocardial sample with the most dense and diffuse leukocytic infiltrate. A score of 0 was assigned to a section in which extravascular neutrophils were not observed. Intermediate scores were assigned based on the varying degree of leukocytic infiltration. All sections were coded so that the pathologist (GDA) was unaware of the experimental treatment.

Depletion of canine polymorphonuclear neutrophils. A purified suspension of canine polymorphonuclear neutrophils was prepared from heparinized whole blood with the Ficoll-hypaque (Sigma Chemical Co.. St. Louis, Mo.) technique of English and Abrams. ${ }^{12}$ Microscopic examination of the purified neutrophil suspension revealed less than $3 \%$ contamination with other cells. A sheep was inoculated by intradermal injection of $10^{\circ}$ neutrophils suspended in complete Freund adjuvant. Ten days later, the sheep received a second challenge of $10^{7}$ neutrophils in incomplete Freund adjuvant. The sheep was bled 20 days after the initial exposure to canine neutrophils. The serum was collected and heat inactivated. Nonimmune serum was obtained by bleeding an uninoculated sheep and heat inactivating the serum. Circulating neutrophil counts in the dog were assessed by total peripheral white cell counts at specific intervals in conjuction with differential counts. Neutrophil antiserum specifically lowered circulating neutrophil counts and did not alter lymphocyte or monocyte concentrations significantly. 


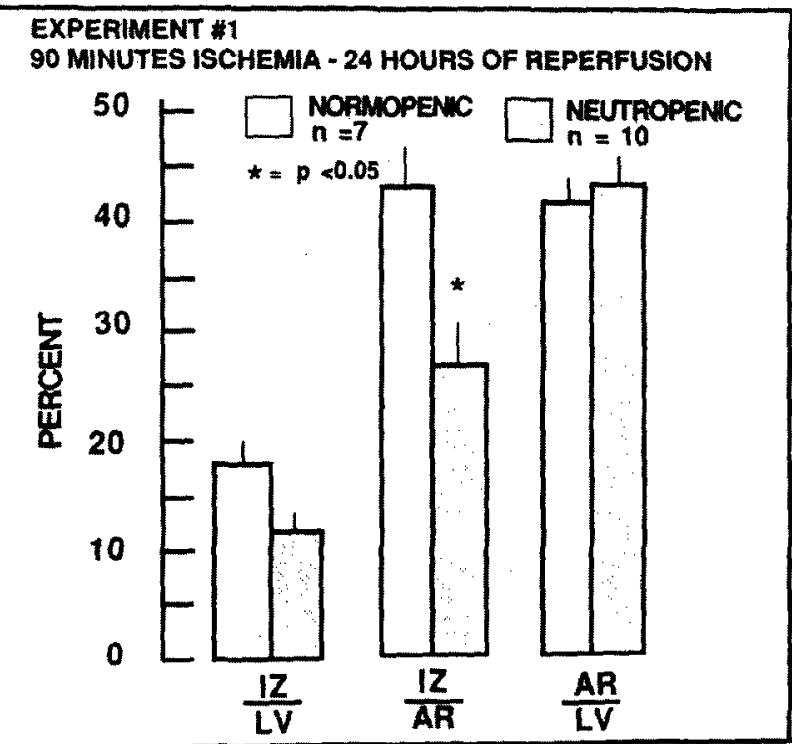

Fig. 5. Summary of results on ultimate infarct size in normopenic and neutropenic dogs subjected to 90 minutes of regional myocardial ischemia (LCCA occlusion) followed by reperfusion for 24 hours. (See text for details.)

Statistics. The mean and standard error of the mean are reported. When more than one group was compared, as in control and occlusion hemodynamic parameters or body weights, one-way analysis of variance was used. When multiple values within groups were evaluated, as in hemodynamic parameters or neutrophil counts at different time points, two-way analysis of variance was used. Statistical significance was analyzed using a suitable multiple comparison test. ${ }^{13}$ Group $t$ statistics were used to compare treated and control groups with regard to parameters measured only once, as in percent of left ventricle infarcted, percent of area at risk infarcted, and percent of total left ventricle at risk. A difference was considered statistically significant at $p<0.05$.

\section{RESULTS}

Thirty-nine dogs were used to evaluate the effect of neutrophil depletion on infarct size resulting from 90 minutes or 4 hours of ischemia. One dog treated with nonimmune serum died of ventricular fibrillation during the course of the 4-hour occlusion/ reperfusion experiment. Two dogs died of unknown causes overnight, one of which was treated with nonimmune serum and one neutropenic dog. Criteria were established before the start of the study to ensure comparable degrees of exposure to regional ischemia among all study groups. For a dog to be included in the statistical analysis, experimental infarct size had to exceed $5 \%$ of the area at risk, and the animal had to show ECG ST segment changes and an area of cyanosis during the period of regional ischemia. Two dogs, one receiving nonimmune serum and one neutrophil depleted, both occluded for 90 minutes, were excluded because of a failure to satisfy the criteria. The remaining 34 dogs were used in the final data analysis: seven nonimmune serum and 10 neutropenic animals undergoing 90 minutes of regional ischemia, and eight nonimmune serum and nine neutropenic dogs subjected to 4 hours of regional ischemia.

The administration of neutrophil antiserum significantly reduced circulating neutrophil counts to $10 \% \pm 3 \%$ in the 90 -minute occlusion experiment and $17 \% \pm 4 \%$ in the 4 -hour occlusion experiment. Results are shown in Fig. 4. In the 90-minute occlusion experiment, significant recovery of circulating neutrophils occurred between 10 hours (the last antiserum injection) and 24 hours (time of euthanasia). In the 4-hour occlusion experiment, a reduction in the circulating neutrophil count was observed at all time points. In both experiments, animals that received nonimmune serum showed increasing neutrophil counts over the course of the experiment. No differences were observed among the four groups with regard to their initial baseline circulating neutrophil counts.

No signiticant differences were observed before coronary occlusion among the four experimental groups with regard to heart rate, mean arterial pressure, the rate times pressure product, LCCA coronary blood flow, or left ventricular end-diastolic pressure (Table I). On occlusion of the LCCA, mean arterial pressure, and the rate times pressure product decreased, while left ventricular end-diastolic pressure increased. Again, no significant difference was observed between the two groups. In experiment No. 1 (90 minutes of ischemia) similar changes in mean arterial pressure, the rate times pressure product, and end-diastolic pressure were observed before reperfusion and at 45 minutes and 2 hours after reperfusion in the groups receiving immune and nonimmune serum. On the next day (experiment No. 1), mean arterial pressure was lower in the neutropenic animals as was the left ventricular end-diastolic pressure when compared with the nonimmune serum control group. Coronary blood flow was not different from control at 45 minutes of reperfusion but was decreased significantly in both groups (neutropenic us. normopenic) before the animals were killed 24 hours after reperfusion.

In experiment No. 2 (4 hours of ischemia) heart rate, mean arterial pressure, and the rate times pressure product were greater in the nonimmune serum-treated group before reperfusion. On reperfusion an increase in heart rate, resulting from 


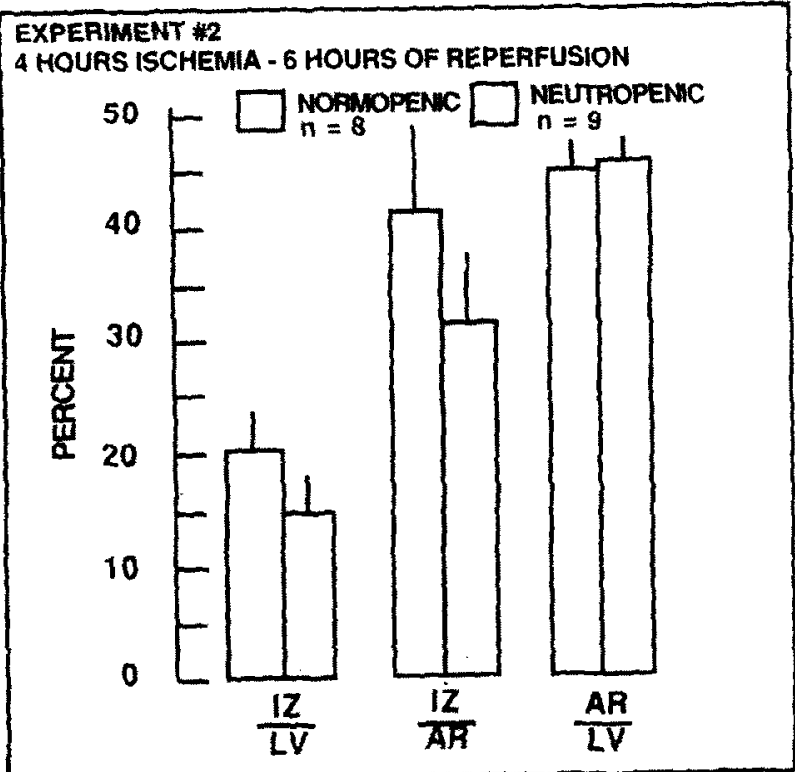

Fig. 6. Summary of results on ultimate infarct size in normopenic and neutropenic dogs subjected to 4 hours of regional myocardial ischemia (LCCA) and 6 hours of reperfusion. (See text for details.)

ventricular tachyarrhythmias in some animals, was observed in both groups. Also, in contrast to experiment No. 1, LCCA blood flow was decreased significantly after 45 minutes of reperfusion in both groups as well as before the time of euthanasia at 6 hours after the induction of neutropenia. In experiment No. 2, left ventricular end-diastolic pressure did not differ between groups before study termination.

The effect of neutropenia on the size of the ultimate myocardial infarct in dogs is shown in Figs. 5 and 6 . Previous results from our laboratory have shown no effect of either sheep ${ }^{10}$ or rabbit ${ }^{1}$ nonimmune serum on infarct size. The induction of neutropenia significantly reduced infarct size in the 90-minute ischemia experiment whether expressed as a percent of total left ventricle $(I Z / L V)$ or percent of the anatomic area at risk (IZ/AR). In the 4-hour ischemia experiment, a tendency toward a reduction in infarct size by in the neutropenic animals did not reach statistical significance. Thus salvage of ischemic myocardium by reduction in the circulating neutrophil count is demonstrated more readily with shorter periods of ischemia before reperfusion.

Histopathologic examination showed ample evidence of irreversible tissue injury in myocardium determined to be infarcted by a failure to react with triphenyltetrazolium. Nonstained myocardium within the area at risk showed extensive necrosis characterized by hyalinization and loss of myofibril-

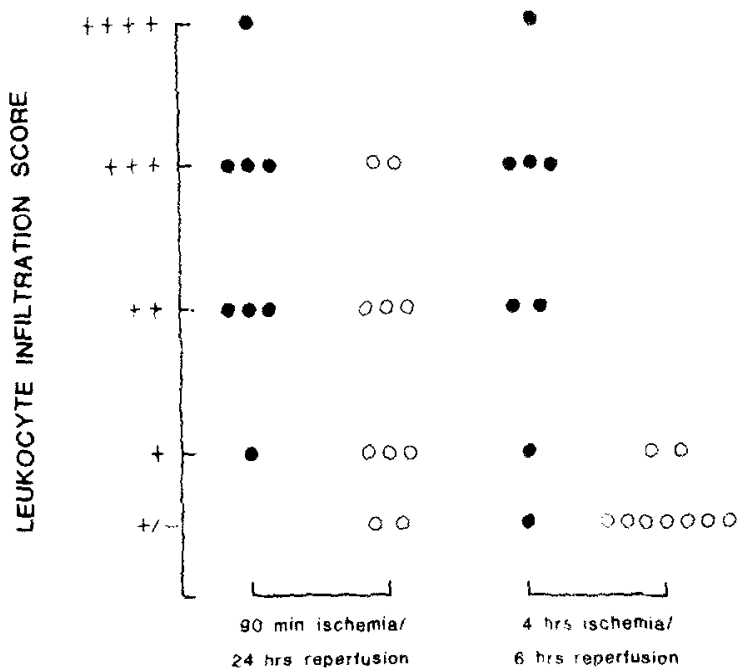

Fig. 7. Results of histopathologic assessment of leukocytic infiltration into infareted myocardium for dogs given nonimmune serum (closed circles) or neutrophil antiserum (open circles). In 90-minute ischemia experiment, significant overlap between groups was evident, which can be attributed to late recovery of circulating neutrophils. In 4-hour ischemia experiment, dogs receiving nonimmune serum had scores ranging from \pm to ++++ (assigned to specimen with most extensive infiltrate). Dogs that received neutrophil antiserum had less prominent neutrophil infiltrates, 7 of 9 dogs being graded $+1-$

lar detail, patchy foci of contraction bands alternating with flocculent cytoplasm, and nuclear changes. Myocardium staining with Evans blue, on the other hand, appeared normal when examined in hematoxylin and eosin-stained sections.

Histologic studies on hematoxylin and eosinstained sections were obtained from hearts of normopenic animals subjected to 4 hours of ischemia and 6 hours of reperfusion showed an extensive infiltration with polymorphonuclear leukocytes. Sections were made through the center of the posterior papillary muscle extending from the endocardial to epicardial regions. Sections taken from comparable regions of hearts of neutropenic dogs made ischemic for 4 hours and reperfused did not show the intensive infiltration with inflammatory cells as seen in the normopenic controls.

Semiquantitative histology was used to grade neutrophil infiltration. The results are summarized in Fig. 7. Infarcts in hearts from dogs that received nonimmune serum in both the 90 -minute and 4hour ischemia experiments showed significant neutrophil infiltration graded $+1-$ to ++++ . Infarcts from neutropenic dogs subjected to 90 minutes of ischemia and 24 hours of reperfusion (experiment No. 1) tended to show a lower neutrophil infiltration 
score. Nevertheless, significant overlap was present. These equivocal results are attributable to the recovery of circulating neutrophil concentrations between the last neutrophil antiserum injection at 10 hours and the time of sacrifice 14 hours later on the next day (Fig 4). Because of the semiquantitative method for assessing the inflammatory response, possible differences were not examined using nonparametric statistics. In contrast, myocardial tissue sections from infarcted regions of hearts of neutropenic dogs subjected to 4 hours of ischemia showed little neutrophil infiltration $(+/-$ to +$)$ in marked contrast to tissue specimens obtained from the hearts of animals in the nonimmune serum group.

\section{DISCUSSION}

The results of the experiments described in the present study emphasize that neutrophil infiltration into infarcted myocardium and release of cytotoxic products by stimulated neutrophils may exacerbate ischemic injury, which is in agrement with an earlier report from our laboratory. Using a rabbit-derived antiserum to reduce canine circulating neutrophils by $77 \% \pm 2 \%$ in a 90 -minute LCCA occlusion with a 6 -hour reperfusion model, Romson et al. ${ }^{1}$ observed that the reduction of circulating polymorphonuclear neutrophils resulted in smaller infarcts without the appearance of significant neutrophil infiltration. The reduction of ultimate infarct size in the 90 minute LCCA occlusion experiment reported herein was identical to that observed by Romson et al. ${ }^{1}$ The ability of neutrophil depletion to produce smaller infarcts appears to be independent of the source of antiserum, whether rabbit ${ }^{1}$ or sheep. The observed similarity in infarct size in the control groups, whether subjected to 6 hours $^{1}$ or 24 hours of reperfusion, suggests that the late recovery $(60 \%)$ in circulating neutrophil counts and the delayed but significant neutrophil infiltration observed at the time of sacrifice did not result in a resumption of myocardial cellular disruptive processes and the ultimate extension of the infarct.

The polymorphonuclear neutrophil initiates the process of organization of injured tissue and recruitment of monocytes to carry out the replacement of necrotic myocardium with fibrous scar tissue. ${ }^{14,15}$ Neutrophil infiltration contributes to demolition of injured tissue followed by macrophage infiltration and resorption of tissue debris. Finally, fibroblasts and capillaries enter the infarcted area and lead to the formation of scar tissue. ${ }^{15}$ The infiltration of neutrophils is an active process stimulated by chemotactic factors within the infarcting myocardium such as C3 anaphylatoxin. ${ }^{16}$ The mechanism of complement activation in ischemic tissue is incom- pletely understood, but studies using cobra venom factor to deplete serum C3 have shown a reduction in ischemic injury in the $\operatorname{dog}^{17,18}$ and baboon ${ }^{19}$ and a reduction of complement localization within the infarct. ${ }^{19}$ Coronary sinus blood in canine myocardial infarction has been noted to possess increased chemotactic activity, ${ }^{18}$ suggesting that ischemically injured myocardium can evoke a chemotactic response.

While acknowledging the role of neutrophils in the healing process, the present study and reports of others showing protective effects of neutropenia ${ }^{1,4,6}$ or complement depletion ${ }^{17-19}$ suggest that the inflammatory response may have a negative impact on potentially viable tissue. Complement deposition in the microvasculature promotes granulocyte adherance, granulocyte aggregation, and endothelial injury. ${ }^{20}$ Aggregation of neutrophils in the ischemic coronary microvasculature has been reported. ${ }^{21}$ Thus, neutrophils may physically contribute to increased ischemic vascular resistance. Stimulated neutrophils trigger the release of arachidonic acid from membrane phospholipids, which may then be converted to vasoactive thromboxane $\mathrm{A}_{2}{ }^{22}$ and to potent chemotactic hydroxyeicosatetraenoic acids (HETES) by specific lipoxygenases ${ }^{23}$ Since radiolabelled microsphere studies examining regional myocardial blood flow during neutrophil depletion have not been conducted, the possibility that myocardial protection was associated with increased regional myocardial blood flow to the risk region is still present. Alternatively, activated neutrophils could contribute to cell death in ischemic myocardium by the production of inflammatory mediators. ${ }^{24}$ Granulocytes stimulated with C5a elaborate cytotoxic oxygen free radicals, ${ }^{25}$ which have been shown to damage endothelial cells in vitro. ${ }^{26} \mathrm{~A}$ study conducted in 1983 used electron spin resonance to show increased oxygen free radicals in ischemic myocardial tissue and coronary sinus blood during ischemia. ${ }^{27}$ Our report showing that the continuous infusion of superoxide dismutase plus catalase could reduce the extent of regional ischemic injury resulting from 90 minutes of occlusion of the LCCA followed by reperfusion supports the concept of free radicalmediated cytotoxicity during reperfusion of the ischemic myocardium. ${ }^{28}$ Additionally, neutrophils can release lysosomal enzymes capable of proteolytic disruption of both viable and irreversibly injured tissue. ${ }^{24}$ In experimental myocardial ischemic injury, the extent of ischemia-induced necrosis is related directly to the duration of coronary artery occlusion. ${ }^{29}$ Regional myocardial blood flow decreases before the advancing wavefront of necrosis. ${ }^{29} \mathrm{By}$ obstructing regional flow through aggregation in the 
microvasculature and by the elaboration of inflammatory mediators, stimulated neutrophils in the early phase of injury after coronary obstruction may represent a mechanism that contributes to the further development of myocardial injury.

The ability of selective interventions to reduce myocardial infarct size may be attributable to an inhibitory action on the neutrophil. Reduction of the extent of irreversible tissue injury by ibuprofen in the dog was associated with a substantial decrease in ${ }^{111} \mathrm{In}$-labeled leukocytes in the infarct region. ${ }^{2}$ Reduction of ultimate infarct size in the cat by ibuprofen also was associated with inhibition of neutrophil infiltration as determined by a post mortem histologic scoring system. ${ }^{5}$ In the same study, pretreatment with aspirin or indomethacin failed to reduce infarct size on reperfusion. In in vitro tests examining the response of feline neutrophils stimulated with FMLP (N-Formyl-L-Methionyl-L-Leucyl-L-Phenyl-alanine), ibuprofen, but not aspirin or indomethacin, inhibited superoxide anion formation and enzyme release. ${ }^{5}$ Effects of the cyclooxygenase-lipoxygenase inhibitor $\mathrm{BW}-755 \mathrm{C}$ to produce smaller myocardial infarcts also may be related to inhibition of neutrophil function. ${ }^{3,4}$ Thus, further studies examining the impact of nonsteroidal antiinflammatory agents and other compounds on neutrophil-mediated inflammation in myocardial ischemia and reperfusion may result in an improved understanding of the cardioprotective effects of these drugs.

In 4-hour occlusion/reperfusion experiments, neutrophil depletion failed to produce significantly smaller infarcts. The reduction in circulating neutrophil concentrations was comparable to that observed in 90-minute studies, ${ }^{1}$ as was the prevention of neutrophil infiltration, ' but large infarcts still occurred. The 4-hour ischemic period constitutes a more severe insult and could not be conducted as an overnight study because of a high rate of mortality. Reperfusion resulted in tachyarrhythmias and in a reduction of LCCA coronary blood flow 45 minutes after reperfusion. While mean infarct size was larger in the 4-hour vs. the 90-minute occlusion experiments, variability in infarct size appeared to be greater in the former series. This observation is supported by the results of Bresnahan et al. ${ }^{30}$ who showed that reperfusion after 5 hours of occlusion reduced infarct size in some animals but resulted in infarct extension in others and was associated with severe hemorrhage. Mean infarct size in the two experiments is not strictly comparable because of different durations of reperfusion that could result in changes in the reference base of myocardial infarction caused by edema, hemorrhage, and other factors. ${ }^{31}$ Large infarcts in neutropenic dogs occluded for 4 hours are consistent with observations reported in a study with verapamil, which produced significant preservation of ischemic myocardium in a 1-hour but not in a 3-hour occlusion reperfusion model. ${ }^{32}$ The severity of tissue injury produced by prolonged ischemia followed by reperfusion may exceed the protection afforded by therapies that appear to be effective with shorter periods of occlusion. Alternatively, the tissue injury attributable to ischemic processes per se may overshadow the inflammatory component. The present study does not intend to contradict that of Ksiezycka and Maroko, ${ }^{6}$ who showed the protective effects of neutrophil depletion in a 6 hour complete occlusion model. It must be remembered that reperfusion, which may have profound effects on infarct size, ${ }^{28,2 \mathrm{~m}}$ was not present in that study.

\section{CONCLUSION}

Reduction in the circulating neutrophil count was associated with the development of smaller myocardial infarcts after 90 minutes of regional ischemia and reperfusion but not with a more prolonged period ( 4 hours) of ischemia. The inhibition of neutrophil infiltration may be an additional approach to the salvage of jeopardized ischemic myocardium and an adjunct to treatment for improvement of ischemic myocardial oxygen balance $^{33}$ but appears to share the limitation that protection is limited by the duration of ischemia. In the 90-minute occlusion experiment, significant recovery of circulating neutrophils in the later phases of the experiment was still associated with smaller infarcts despite significant neutrophil infiltration. Further studies are required to define the critical period in which neutrophil depletion is protective and to determine the extent to which neutrophils can then recover and participate in the organization and healing of the infarct. Impaired infarct healing after antiinflammatory therapy with high dose methylprednisolone ${ }^{34}$ and ibuprofen ${ }^{35}$ has been described. Efforts to salvage ischemic myocardium by inhibiting neutrophil infiltration and activation will be of experimental interest until longterm benefits have been documented

We wish to acknowledge the excellent technical assistance of Mr. John Liddicoat and Mr. Edward Driscoll and express appreciation for secretarial help from Ms. Pat Quiel and Miss Kim Kanitz

\section{REFERENCES}

1. Romson JL, Hook BG, Kunkel SL, Abrams GD, Schork MA, Lucchesi BR: Reduction of the extent of ischemic myocardial injury by neutrophil depletion in the dog. Circulation $1983 ; 67: 1016$. 
2. Romson JL, Hook BG, Rigot VM, Schork MA, Swanson DP, Lucchesi BR: The effect of ibuprofen on accumulation of "Indium-labelled platelets and leukocytes in experimental myocardial infarction. Circulation 1982;66:1002.

3. Jolly SR, Lucchesi BR: Effect of BW-755C in an occlusion reperfusion model of ischemic myocardial injury. Am Heart $\mathrm{J}$ $1983 ; 106: 8$.

4. Mullane KM, Read N, Salmon JA, Moncada S: Role of leukocytes in acute myocardial infarction in anesthetized dogs: relationship to salvage by antiinflammatory drugs. $J$ Pharmacol Exp Ther 1984;228:510.

5. Flynn PJ, Becker WK, Vercellotte GM, et al: Ibuprofen inhibits granulocyte responses to inflammatory mediators, a proposed mechanism for reduction of experimental myocardial infarct size. Inflammation 1984;8:33.

6. Ksiezycka E, Maroko PR: Reduction by antineutrophil serum of experimental infarct size. Clin Res 1983;31:666A

7. Lucchesi BR, Burmeister WE, Lomas TE, Abrams GD: Ischemic changes in the canine heart as affected by the dimethyl analogue of propranolol UM-272 (SC-27761). J Pharmacol Exp Ther 1978;199:310.

8. Hosko MJ, Gross GJ, Warltier DC: Technique for precise graded arterial stenosis and occlusion. Basic Res Cardiol 1977:72:651.

9. Boudoulas H. Karayannacos PE, Lewis RP, Kakos GS, Kilman JW, Vasko JS: Potential effect of lidocaine on ischemic myocardial injury: experimental and clinical observations. J Surg Res 1978;24:469.

10. Jolly SR, Schumacher WA, Kunkel SL, Abrams GD, Liddicoat J, Lucchesi BR: Platelet depletion in experimental myocardial infarction. Basic Res Cardiol 1985;80:269.

11. Bush LR, Romson JL, Ash JL, Lucchesi BR: Effects of diltiazem on extent of ultimate myocardial injury resulting from temporary coronary artery occlusion in dogs. J Cardiovasc Pharmacol 1982;4:285.

12. English D. Abrams S: Single-step separation of red blood cells, granulocytes and mononuclear cells on discontinuous density gradients of Ficoll-hypaque. J Immunol Methods 1974;5:249.

13. Duncan DB: Multiple range and multiple $f$-tests. Biometrics $1955 ; 11: 1$

14. Mallory G, White P, Salcedo-Salgar J: The speed of healing of myocardial infarction. A study of the pathologic anatomy in seventy-two cases. Am Heart J 1939;18:647.

15. Lautsch E: Morphologic factors of clinical significance in myocardial infarction, a review. Tex Rep Biol Med 1979;39:371.

16. Hill JH, Ward PA: The phlogistic role of C3 leukotactic fragments in myocardial infarcts of rats. J Exp Med 1971; 133:885.

17. Maroko RR, Carpenter CB, Chiariello M, et al: Reduction by cobra venom factor of myocardial necrosis after coronary artery occlusion. J Clin Invest 1978;61:661.

18. Pinckard RN, O'Rourke RA, Crawford MH, et al: Complement localization and mediation of ischemic injury in baboon myocardium. J Clin Invest 1980;66:1050.

19. Hartmann JR, Robinson JA, Gunnar RM: Chemotactic activity in the coronary sinus after experimental myocardial infarction: effects of pharmacologic interventions on ischemic injury. Am J Cardiol 1977;40:550.
20. ('raddock PR, Hammerschmidt DE, Moldow CF, Yamada O, Jacob HS: Granulocyte aggregation as manifestation of membrane interactions with complement: possible role in leukocyte margination, microvascular occlusion, and endo. thelial damagc. Scmin Hematol 1979;16:140.

21. Engler RL, Schmid-Schonbein GW, Pavalec RS: Leukocyte capillary plugging in myocardial ischemia and reperfusion in the dog. Am J Pathol 1983;111:98.

22. Goldstein IM, Malmsten CL, Samuelsson B, Weissman (i: Prostaglandins, thromboxanes and polymorphonuclear leukocytes. Mediation and modulation of inflammation. Inflam mation 1977;2:309.

23. Snyderman R, Goetzl E: Molecular and cellular mechanisms of leukocyte chemotoaxis. Science 1981;213:830.

24. Weissman G, Smolen J, Korchak H: Release of inflammatory mediators from stimulated neutrophils. $N$ Engl i Med 1980;303:27.

25. Fantone JC, Ward PA: Role of oxygen derived tree radicals and metabolites in leukocyte-dependent inflammatory reac: tions. Am J Pathol 1982;107:397.

26. Sacks T, Moldow CF, Craddock PR, Bowers 'TK, Jacob HS: Oxygen radicals mediate endothelial cell damage hy comple. ment-stimulated granulocytes. An in vitro model of immune vascular damage. I Clin Invest 1978;61:11ti1.

27. Rao PS, Cohen MV, Mueller HS: Production of free radicals and lipid peroxides in early experimental mvocardial ische'mia. J Mol Cell Cardiol 1983;15:713.

28. Jolly SR, Kane WJ, Bailie MB. Abrams (il), Lucchesi BR: A canine model of reperfusion injury, its reduction by the combined administration of superoxide dismutase and catalase. Circ Res 1984;54:277.

29. Reimer K, Jennings $R$ : The "wavefront phenomenon" of myocardial ischemic cell death. II. T'ransmural progression of necrosis within the framework of ischemic bed size (myocar. dium at risk) and collateral flow. Lab Invest 1979;40:633.

30. Bresnahan GF, Roberts R, Shell WE, Russ J, Sobel BE: Deleterious effects due to hemorrhage after myocardial reperfusion. Am J Cardiol 1974;33:82.

31. Reimer KA, Jennings RB: The changing anatomic reference: base of evolving myocardial infarction. Underestimation of myocardial collateral blood flow and overestimation of exper imental anatomic infarct size due to tissue edema, hemor rhage and acute inflammation. Circulation 1979;60:866.

32. Reimer KA, Jennings RP: Verapamil in two reperfusion models of myocardial infarction: temporary protection of severely ischemic myocardium without limitation of ultimate. infarct size. Lab Invest (In press).

33. Romson JL, Jolly SR, Lucchesi BR: Protection of ischemic myocardium by pharmacologic inhibition of leukocyte func tion. Cardiovasc Rev Rep 1984;5:690.

34. Kloner R, Fishbein M, Lew H, Maroko fs, Braunwald E Mummification of the infareted myocardinm by high dose steroids. Circulation 1978;57:56.

35. Brown EJ, Kloner RA. Schoen F.J, Hammerman H, Dale $S$ Braunwald E: Scar thinning due to ibuprofen administration following experimental myocardial infarction. Am J Cardiol 1983;51:877. 